\title{
On General Physical Principles of Biological Evolution
}

\author{
G. P. Gladyshev \\ Russian Academy of Arts and N. N. Semenov Institute of Chemical Physics of Russian Academy of \\ Sciences, Moscow, Kosygina, 4.
}

\begin{abstract}
This short overview presents the main achievements of the physical theory of biological evolution from the viewpoint of hierarchical thermodynamics which was createdby the author on the foundation of Gibbs thermodynamics.

The origin and evolution of life is the process of origin and development of relatively stable dynamic hierarchical structures of matter in the universe areas, which are characterized by physical properties and chemical composition suitable for the existence of these structures.

Nature seeks maximum relative stability of structures at all hierarchical levels of matter. Hierarchical thermodynamics is the foundation of Darwinism. The theory explains and predicts many facts and phenomena.
\end{abstract}

Keywords: biological evolution; life, hierarchies; thermodynamics; hierarchical thermodynamics; stability; Darwinism; aging; principle of substance stability.

\section{Epigraphs:}

"One of the principal objects of theoretical research in any department of knowledge is to find the point of view from which the subject appears in its greatest simplicity."

J. Willard Gibbs

“...nothing at all takes place in the universe in which some rule of maximum or minimum does not appear ..."

Leonhard Euler

\section{INTRODUCTION}

First of all, in order to avoid confusion, it should be noted that this article shows individual results concerning hierarchical thermodynamics. This thermodynamics is the quasi-equilibrium linear dynamic thermodynamics created by theauthor [1-8]. The author's hierarchical thermodynamics has nothing to do with thermodynamics of systems far from equilibrium of I. Prigogine $[9,10]$, which (the theory) does not apply to the origin and the evolutionary development of the living world, and which, in my opinion, is a misguided theory. This theory in the context of biological evolution has only historical significance.However, it should be noted that the dynamic approach to the study of natural real dissipative structures has an attractive future.

\section{MethodS}

The author created the thermodynamic theory of origin of life, its evolution, and aging of living beings and laid the foundations of hierarchical thermodynamics. The author used mainly the deductive method of J.W. Gibbs.

\section{RESUlTS AND DisCUSSION}

\subsection{The Hierarchic Thermodynamic Theory of Evolution and Extended Darwinism}

Modern thermodynamics investigates spontaneous and nonspontaneous evolutionary processes in biological systems. The hierarchical thermodynamics is the foundation of extended Darwinism [4-8].

Spontaneous transformation of structures within each hierarchy system proceeds according to the second law of thermodynamics. These processes are predictable. 


\section{G. P. Gladyshev}

Nonspontaneous transformation of structures within the framework of each hierarchy is initiated by actions of the environment. These transformations as a result of changing environmental influences can be unpredictable.

The environment of systems of each hierarchy is structures of other hierarchies. However, in the absence of revolutionary changes and with relatively small fluctuations of environmental conditions that occur in the adaptive area, the general evolutional trend of the second law takes place. In this case, the driving force of evolution processes first of all thermodynamics of spontaneous transformations in biological systems: nature searches minimums of the specific free energies of formation of structures (maximum of stability) at all hierarchical levels.

It is very important to draw attention to the fact that any theory of biological evolution (and also the theory of aging of living organisms) should be based on thermodynamic phenomena.

According to the author's hierarchical thermodynamic theory of evolution, the origin of life and evolution proceeds in accordance with the second law (the spontaneous processes in chemical and biological systems) against the background of the changing environment (nonspontaneous processes in systems that are initiated by the environment).

From the point of view of spontaneous processes, the driving force of biological evolution and development is the principle of substance stability. The existence of a high order in liquid-crystal structures of the living world suggests that the principle of substance stability acts not only on all the major adjacent hierarchical levels, but also within each hierarchical level and sub-level. A proof of this statement is the well-known identity of the majority of molecular and morphological structures of identical twins.

All processes of evolution are taken into account by the advanced hierarchical generalized Gibbs equation, the generalized equation of the first and second laws of thermodynamics [5-7]:

$$
d G^{*}=\sum_{i} d G_{i}^{*}=-\sum_{i} S_{i} d T_{i}+\sum_{i} V_{i} d p_{i}-\sum_{i} \sum_{k_{i}} x_{k_{i}} d X_{k_{i}}+\sum_{i} \sum_{k_{i}} \mu_{k_{i}} d m_{k_{i}}
$$

Where $G$ is the Gibbs free energy; $T$ is the temperature; $S$ is the entropy; $V$ is the volume; $\mathrm{p}$ is the pressure; $X$ is any generalized force except pressure; $x$ is any generalized coordinate except volume; $\mu$ is the chemical (evolutionary) potential; $m$ is the mass of the $k$-substance; the work performed by the system is negative. The subscript $i$ pertains to the specific evolution, and $k$ to the component $i$ evolution. The superscript $*$ means that behavior of a quasi-equilibrium complex system is considered.

If the action of the environment is outside the area of adaptive existence of organisms (living systems), there is a gap in the evolutionary spiral. This may lead to disappearance of life. For example, such a revolutionary (non-evolutionary) phenomenon was observed in the extinction of the dinosaurs.

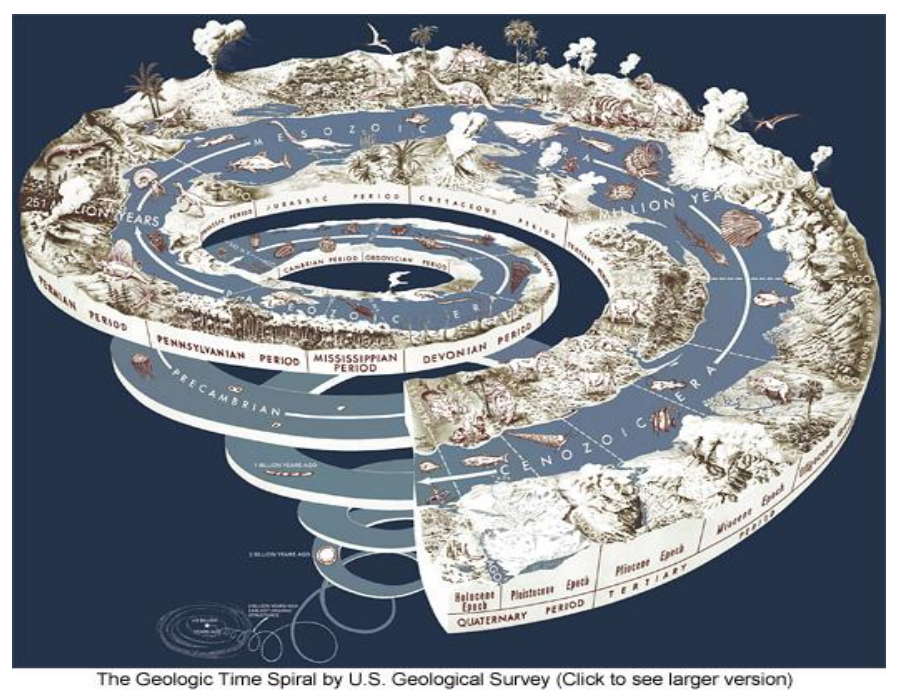

Fig1. Spiral of evolution. Hierarchical thermodynamics converts evolutionary biology from "the science in pictures" into an exact science. 
The thermodynamic theory of evolution claims that Darwin and Lamarck are both right. The reason is that "transformations" of Darwin and Lamarck occur at substantially different times (i.e. in different time scales).

The origin of life and its evolution are linked to the advent of polyhierarchical structures that develop in accordance with the principle of substance stability. This thermodynamic principle applies at all levels and sub-levels of living matter. This is the general principle of life existence.

\subsection{How to Develop the Theory of Darwin}

Darwin's theory is a general descriptive theory. It is based, first of all, on the common notions of "variation and selection", which, incidentally, act at all hierarchical levels of living systems. To describe evolution from the viewpoint of these positions it is necessary to apply this Darwinian approach separately to the individual structural hierarchy of living systems and organisms. In other words, we need to study the evolution of objects of the same type (molecules, organelles, cells, organisms, populations, and so on.) Investigation of behavior of no identical objects from the scientific position has no physical meaning. Darwin's theory is not an exact theory. This theory may only indicate "the general thermodynamic mechanism" (thermodynamic tendency) of individual processes. Application of demands that we use to study simple systems to Darwin's theory does not make sense. General concepts of" variation and selection" have proven themselves in their application to the study of similar systems and processes, for example, in relation to the chemical evolution. The discussion of Darwin's theory only from the generalized biology position, in my opinion, cannot contribute to the development of the theory. Hierarchical thermodynamics is the foundation of the expanded theory of Darwin.

We cannot use scientific methods without dissecting complex phenomena into simple components. Scientists of exact physical sciences know about this.

\subsection{Explanations and Predictions of Some Facts}

Hierarchical thermodynamics predicted and substantiated many facts and phenomena [1-8]. Primarily, the theory proved that changes of chemical compositions of living beings (and also compositions of structures of others hierarchies) had a thermodynamic origin. It turned out that the trend of reducing the amount of water in living organisms in ontogeny, phylogeny, and evolution of the living world is first and foremost the result of supramolecular thermodynamics.

The theory explained the significant difference of the chemical element composition of flora and fauna. In accordance with the guide thermodynamic force, significant enrichment of animals by nitrogen, phosphorus, and to a small extent by sulfur is observed in the transition from plants to animals. Despite the lack of information about the absolute stability of chemical substances, the theory predicted the increase in the concentration of compounds with low stability (the compounds which have $\mathbf{C}-\mathbf{N}, \mathbf{O}-\mathbf{N}, \mathbf{H}-\mathbf{N}$ bonds and similar chemical bonds of $\mathbf{P}$ and $\mathbf{S}$ ) in living organisms during evolution.

Also, a consequence of the action of thermodynamics, as predicted by the author, is enrichment of living organisms during evolution by heavy elements and by isotopes. A good prediction of this trend is the fact that the iodine concentration is increased in the process of evolution of living beings.

It has been established that the principle of the stability of the substance of the author is the driving force behind the origin of life and its development, which manifests itself in the emergence and improvement of the polyhierarchical structure and design of the forms of biological objects in their environments. The principle of substance stability (the principle of feedback) from the viewpoint of thermodynamics proved and predicted existence of thermodynamic transfer of information from higher hierarchic to lower hierarchic biological structures, for example, from populations to DNA structures. The author managed to demonstrate applicability of the principle of substance stability to explaining the maintenance of a high concentration of adenosine triphosphate (ATP), and other power-consuming chemically unstable metabolites in the tissues of organisms. Of particular note is relatively low chemical stability of explosives which was predicted by the principle.

The theory explained the action (which stems from centuries) of the principle "divide and rule", as well as some well-known laws of social development. 


\section{G. P. Gladyshev}

The author of hierarchical thermodynamics believes that history is predictable. It was also proved that Darwin and Lamarck were both right: the evolutionary theories of these creators describe the development of living systems on disparate time scales.

The principle was experimentally proved in the study of many inorganic and organic systems.

The thermodynamic theory predicted composition of a number of diets for maintaining a healthy life and for increasing longevity. Some diets, previously identified only on a trial basis, now are more widely applicable in practice. Thermodynamics substantiated the existence of "nutrient" and other similar small molecules and explained their impact on the expression of genes at the epigenetic level.

There is an interesting prediction of hierarchical thermodynamics: the theory predicted the existence of a single common "molecular code of life" for our universe. This prediction is unproven. However, this conclusion of hierarchical thermodynamics agrees with the ideas and results of Fred Hoyle and Chandra Wickramasinghe [11].

Currently, some researchers began to consider hierarchical thermodynamics as the foundation of extended Darwinism.

On a number of Internet sites, we can find examples of the use of the theory in different areas of knowledge.

\section{Conclusions}

The origin and evolution of life is the process of origin and development of relatively stable dynamic hierarchical structures of matter. Nature tends to the maximums of relative stability of structures at all hierarchical levels and sublevels of living systems. Hierarchical thermodynamics is the physical foundation of extended Darwinism.

The thermodynamic theory explains and predicts many facts and phenomena.

The origin of life and its evolution are linked to the advent of hierarchical chemical and biological structures that develop in accordance with the principle of substance stability, the general physical principle of life existence.

\section{Application}

\subsection{Misunderstandings in Ideas about Entropy}

Many discrepancies in understanding the problems of life and evolution from the standpoint of physics and physical chemistry are typically associated with misconceptions in understanding entropy [4-7]. The term "entropy" was coined by Rudolf Clausius. Following his model of the world (universe), he stated: "The energy of the world is constant. The entropy of the world tends to the maximum". Later this statement was chosen by J.W. Gibbs as an epigraph to his paper "On the Equilibrium of Heterogeneous Substances". These scientists have given this statement in relation to their model of the universe. This model corresponds to a simple isolated system of ideal gas, i.e., an isolated system of ideal gas, in which the energy and volume are constant and only the work of expansion is performed. The entropy of such a system can only increase!

It should be noted that when we speak about the ideal model that would correspond to the real universe, it would be necessary to accept the unreal assumption that any form of energy in the real universe will be transformed into thermal energy. Only in this case, and under additional unrealistic assumptions, the real universe "would turn" into the Clausius-Gibbs model of the ideal system.

However, science amateurs applied representations of simple systems to systems of other types, in which interactions takes place between particles of different nature (interactions of molecules or other objects of different hierarchies) and to systems which interact with the environment. Some scientists, who are not professionals in the relevant fields of knowledge, did not escape such errors. This led to unimaginable confusion and slowed down the development of science for more than a century. There are thousands of publications in scientific journals and popular literature containing marked misunderstandings. To these were added incorrect ideas on negentropy and on dissipative structures in the living world and the false identification of "the information entropy" with the thermodynamic entropy. 
The origin of life and its evolution can be easily explained from the standpoint of hierarchical nearequilibrium thermodynamics of complex dynamic systems. This thermodynamics is established on a solid foundation of equilibrium thermodynamics, thermodynamics of R. Clausius, J.W. Gibbs, and other great scientists.

\subsection{Difference between the Thermodynamic and the Dynamic Self-Organization}

Many researchers do not distinguish processes of formation of relatively thermodynamically stable supramolecular structures (e.g., living tissues) and temporarily existing dynamical molecular dissipative structures. Supramolecular structures of living things arise as a result of self-assembly - the near-equilibrium thermodynamic self-organization. This process resembles formation of crystals in the conditions close to equilibrium.

The dissipative structures arise as a result of the far-from-equilibrium dynamic self-organization in systems which are far from equilibrium. This self-organization takes place as a result of the sharp mixing of reagents. For example, this case is realized in the Belousov - Zhabotinskii reaction and in the similar processes previously described by K. Denbigh and other scientists. Similar oscillatory processes occur in living organisms. For example, the fluctuations of concentrations of sugar are easily observed in the blood of animals that rapidly "simultaneously" eat up large amounts of this substance. Here we are dealing with the non-stationary processes, which we can investigate using kinetic parameters only. It should be noted that the dissipative structures in fluid flows were described earlier in the works of Leonardo da Vinci.

"The self-organization of Prigogine" is a dynamic self-organization which is not described using the state functions, i.e., functions that have total differentials. So, "the entropy of Prigogine" used to describe systems far from equilibrium is a kind of kinetic function that cannot be calculated. The theory of dissipative structures of Prigogine from the perspective of biological evolution and aging of organisms does not apply to living systems and is only of historical interest. However, dynamic approaches to the study of real dissipative structures in nature have an attractive future.

\subsection{On the Entropy Production of Living Systems}

Some researchers remember Boltzmann's statement about the struggle of the living creatures behind the entropy. It is often believed that life is associated with an increase in negative entropy (negentropy) of living organisms [4-6]. However, it is not clear at what hierarchical level these processes take place. For example, we can talk about the production of entropy at the molecular or supramolecular level. In these cases, the value of entropy production can be both negative and positive. Entropy can decrease or increase. The enthalpy-driven processes and the entropy-driven processes are well investigated in biology[12].Note that the entropy production in the system far from equilibrium cannot be calculated.

In any case, the change in entropy in living systems during their aging and evolution does not determine the direction of these processes. In order to avoid contradictions, it is believed that the total entropy production of the living systems and the universe is positive in value. However, there is no evidence that the entropy of the real universe must increase. The presented considerations could only refer to the model of an ideal universe as a simple thermodynamic model. The real universe can be viewed only as a complex thermodynamic system, i.e. a system in which different types of work are performed, but not only the work of expansion. It can be argued that the approach from the viewpoint of the change of entropy in the study of the origin of life and its evolution is a hopeless approach.

Living systems can be regarded as quasi-closed systems, in which quasi-equilibrium processes take place during certain scales of times. The direction of these processes can be established using the method of hierarchical thermodynamics, which was created on the foundation of phenomenological thermodynamics by J. W. Gibbs [1-7].

\subsection{On Standard points in Hierarchical Thermodynamics}

It is important to remember that hierarchical thermodynamic approaches are often associated with the use of "floating standard points". This can cause concern professionals of thermodynamics. However according to experimental data these standard points can be used to identify trends of changing of chemical and supramolecular stability of systems of variable compositions during evolution and aging of living beings. This suggests that basic chemical elements of living systems can be considered as "the same-type elements" from the viewpoint of thermodynamics. 


\section{REFERENCES}

[1] Gladyshev Georgi P., On the Thermodynamics of Biological Evolution, Journal of Theoretical Biology, Vol. 75, Issue 4, Dec 21,1978, pp. 425-441 (Preprint, Chernogolovka, Institute of Chem. Phys. Academy of Science of USSR, May, 1977, p. 46).

[2] Gladyshev Georgi P., Thermodynamics Theory of the Evolution of Living Beings, Commack, New York: Nova Science Publishers, Inc., 1997. 142 P. In Russian: Гладышев Г.П., Термодинамическая теория эволюции живых существ, М:"Луч", 1996.-86c. http://www. statemaster,com/encyclopedia/History-of-thermodynamics

[3] Гладышев Г.П. Супрамолекулярная термодинамика - Ключ к осознанию явления жизни. Что такое жизнь с точки зрения физико-химика. Издание второе, М - Ижевск. ISBN: 59397-21982 (2003).

[4] Gladyshev G. P., Natural Selection and Thermodynamics of Biological Evolution, Natural Science, 7, pp. 117-126(2015). http://dx.doi.org/10.4236/ns.2015.73013

[5] Gladyshev Georgi P., Thermodynamics of Aging and Heredity, Natural Science, 7, No 5, pp. 270-286(2015). http://www.scirp.org/Journal/PaperInformation.aspx?PaperID=56613

[6] Gladyshev G.P., The Thermodynamic Theory of Evolution and Ageing, Advances in Gerontology, 4, 109-118(2014). http://dx.doi.org/10.1134/S2079057014020064

[7] Гладышев Г. П. Иерархическая термодинамика и эволюция химической и биологической материи. (Gladyshev G.P., Hierarchical thermodynamics and evolution of chemical and biological matter). The scientific heritage: Budapest, Hungary.Vol. 1, № 3 (3), pp.102121(2016).https://www.researchgate.net/publication/309533110Hierarchical_thermodynamics_a nd_evolution_of_chemical_and_biological_matter

[8] Gladyshev Georgi, Thermodynamics Optimizes the Physiology of Life, p. 152 - 163.In: Philosophy \& Cosmology,v. 12(2014), pp. 152-163. ISSN 2307-3705. http://ispcjournal.org/ journals/2014/PhC_2014.pdf

[9] Shu-Kun Lin, Diversity and Entropy, Editorial, Entropy, 1(1), pp. 1-3(1999). http:// www. mdpi.com/1099-4300/1/1/1/htm doi:10.3390/e1010001

[10] Thims Libb, Georgi Gladyshev - Thermodynamics of evolution (2010).https://www.youtube. $\mathrm{com} /$ watch? $=$ CYr1G5TZO50

[11] Wickramasinghe Chandra, The Dawn of a new Enlightenment (2017).http://www.island.lk/ index.php?page_cat $=$ articledetails $\&$ page $=$ articledetails\&code_title $=158819$

[12] Lauffar M. A., Entropy-Driven processes in biology, B., N.Y.: Springer (1975). 